\title{
Análise de benzeno, tolueno, etilbenzeno e xilenos em solos por headspace e cromatografia gasosa/detector de ionização de chama
}

\author{
Analysis of benzene, toluene, ethylbenzene and xylenes in soils by \\ headspace and gas chromatography/flame ionization detector
}

\author{
Rafael Augusto Melquiades ${ }^{1}$; Ilza Lobo²; Carmen L. B. Guedes²; \\ Jurandir Pereira Pinto ${ }^{3}$
}

\begin{abstract}
Resumo
Os constituintes da gasolina benzeno, tolueno, etilbenzeno e xilenos (BTEX) são freqüentemente encontrados em solos devido a vazamentos de tanques de estocagem de combustíveis e apresentam toxicidade crônica. Neste trabalho foi desenvolvida e validada uma metodologia de análise de BTEX em solo, usando a técnica de cromatografia gasosa/detector de ionização de chama e headspace estático. A recuperação de BTEX em amostras de solo foi avaliada utilizando solos com diferentes texturas (arenoso e argiloso). O método de análise mostrou boa resolução, em um baixo tempo de análise (menos de 30 minutos). Os limite de quantificação de $0,05 \mathrm{mg} \mathrm{Kg}^{-1}$ solo para benzeno, tolueno, etilbenzeno e xilenos encontram-se abaixo dos valores orientadores, que variam de 0,15 a $95 \mathrm{mg} \mathrm{Kg}^{-1}$ solo, estabelecidos para determinar a qualidade do solo. Verificou-se que a metodologia permite a aplicação deste método para a análise de BTEX em amostras de solos com o objetivo de identificar o passivo ambiental de postos de combustíveis.
\end{abstract}

Palavras-chave: BTEX. Headspace. CG-DIC. Solo.

\begin{abstract}
The constituents of gasoline: benzene, toluene, ethylbenzene and xylenes (BTEX) are frequently found in soils due to leaks in fuel storage tanks and they present chronic toxicity. In this work it was developed and validated a methodology of BTEX analysis in soil by gas chromatography/ flame ionization detector and static headspace. The recovery of BTEX in soil samples was evaluated using soils with different textures (sandy and loamy). The analysis method showed good resolution, in a low time of analysis (less than 30 minutes). Limits of quantification of 0.05 $\mathrm{mg} \mathrm{Kg}^{-1}$ soil for benzene, toluene, ethylbenzene and xylenes are below the guiding values that range from 0.15 to $95 \mathrm{mg} \mathrm{Kg}^{-1}$ soil, established to determine soil quality. It was verified that the methodology enables the use of this method for BTEX analysis of soil samples for passive environmental identification of gas stations.
\end{abstract}

Key words: BTEX. Headspace. GC/FID. Soil.

\footnotetext{
${ }^{1}$ Estudante do Curso de Química - UEL - Londrina.

${ }^{2}$ Docentes do Departamento de Química - CCE - Universidade Estadual de Londrina, Londrina. E-mail: lobo@uel.br.

${ }^{3}$ Técnico do Departamento de Química - CCE - Universidade Estadual de Londrina, Londrina.
} 


\section{Introdução}

Nos últimos anos, com o aumento da população e da atividade industrial, intensificaram-se as preocupações com relação à qualidade da água. A contaminação de águas subterrâneas e solos por produtos orgânicos, como conseqüência de derramamentos, acidentes, ou resíduos industriais, representa hoje sérios problemas à saúde pública. Contaminação por benzeno, etilbenzeno, tolueno e isômeros de xileno (BTEX), estão associados a produtos derivados do petróleo, como por exemplo, a gasolina que apresenta cerca de 10 a $59 \%$ de compostos aromáticos (EZQUERRO et al., 2004). Os maiores problemas das contaminações por derivados de petróleo são atribuídos aos hidrocarbonetos monoaromáticos (BTEX), que são os constituintes mais solúveis e mais móveis da fração da gasolina, sendo os primeiros a atingir o lençol freático (ARAMBARRI et al., 2004). Estes compostos são poderosos depressores do sistema nervoso central, e apresentam toxicidade crônica, mesmo em pequenas concentrações (da ordem de $\mathrm{ppb}$ ), sendo o benzeno o mais tóxico e comprovadamente carcinogênico. O comportamento de derivados de petróleo no solo é governado por diferentes fatores que incluem características do solo, como propriedades químicas, físicas e mineralógicas e fatores ambientais como temperatura e precipitação (SEMPLE; REID; FERMOR, 2001). No solo, os componentes voláteis, como BTEX, podem ser volatilizados para a fase gasosa e, a seguir, podem ser perdidos para a atmosfera, ou adsorvidos nos sólidos dos solos, ou dissolvidos na solução do solo ou na água subterrânea (FINE; GRABER; YARON, 1997). Com o objetivo de proteger a água subterrânea, os órgãos ambientais estão exigindo a avaliação do passivo ambiental de postos de combustíveis, na qual devem ser monitorados e quantificados os hidrocarbonetos aromáticos no solo, incluindo benzeno, tolueno, etilbenzeno e xilenos (Figura 1). Técnicas tais como headspace dinâmico (purge and trap), micro extração em fase sólida (SPME) e micro extração com solvente (SME) têm sido desenvolvidas para melhorar a eficiência da extração para compostos orgânicos voláteis e semi-voláteis de amostras líquidas e sólidas (MARTINEZ et al., 2002). Estas técnicas requerem equipamentos caros e, no caso da SPME, a fibra degrada-se com o uso contínuo e tem um tempo de vida limitado, e do mesmo modo, cabe levar em conta os tipos de fases estacionárias limitadas para as fibras (MOHAMMADI; ALIZADEH, 2006). A técnica de headspace estático é relativamente simples e de fácil operação. Nela a amostra no headspace é preparada em um frasco que, além da amostra, contém o solvente de diluição e a solução modificadora (Figura 2). Neste contexto o objetivo deste trabalho foi desenvolver e validar um método de análise de BTEX pela técnica de cromatografia gasosa e headspace estático, usando solos de diferentes texturas (argiloso e arenoso). Além disso, buscou-se aplicar esta técnica em solos contaminados provenientes de postos de combustíveis.

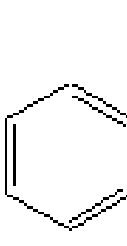

Benreno<smiles>Cc1ccccc1</smiles>

Toheno<smiles>CCc1ccccc1</smiles>

Etibenzero<smiles>Cc1ccccc1C</smiles>

o-Xileno

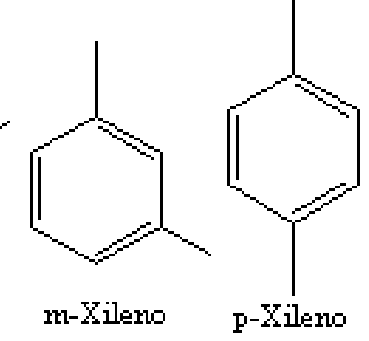

Figura 1. Estrutura química dos hidrocarbonetos monoaromáticos benzeno, tolueno, etilbenzeno e xilenos. 


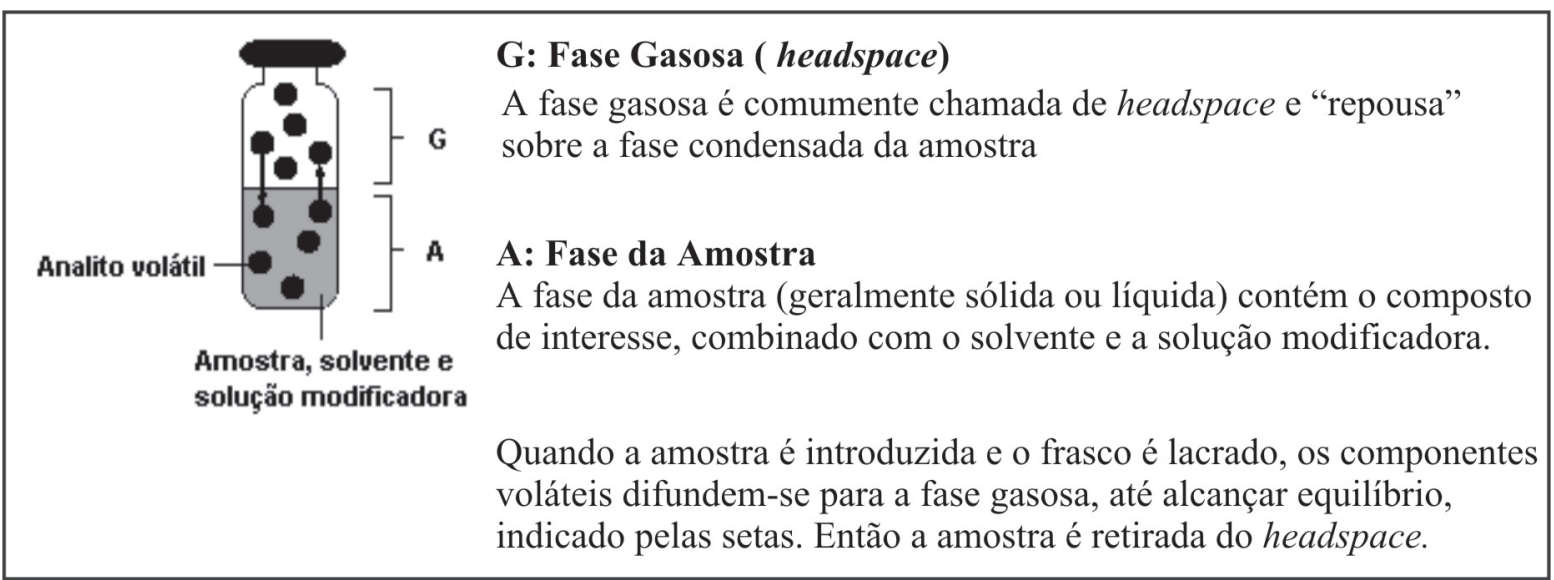

Figura 2. Fases do frasco do headspace.

Fonte: Restek (2004).

\section{Materiais e Métodos \\ Solução Modificadora}

Uma solução aquosa a $36 \%(\mathrm{~m} / \mathrm{v})$ foi preparada dissolvendo $180 \mathrm{~g}$ de $\mathrm{NaCl}$ (p.a, SYNTH) em balão volumétrico de $500 \mathrm{~mL}$, com água deionizada. $\mathrm{O} \mathrm{pH}$ dessa solução foi ajustado a 2,0 pela adição de ácido fosfórico concentrado a $85 \%$ (SYNTH).

\section{Condições Cromatográficas}

A separação dos componentes BTEX foi otimizada por CG-DIC (SHIMADZU 17A) usando uma coluna: DB1 (100\% Siloxano), 30m x 0,25mm x $0,25 \mathrm{ìm}$ (J\&W SCIENTIFIC). Estabeleceu-se a melhor condição: temperatura inicial $40,0{ }^{\circ} \mathrm{C}$ por 6 minutos e rampa de aquecimento $4,0{ }^{\circ} \mathrm{C} \mathrm{min}^{-1}$ até $120{ }^{\circ} \mathrm{C}$ e $40,0{ }^{\circ} \mathrm{C} / \mathrm{min}$ até $200{ }^{\circ} \mathrm{C}$. Injetor a $200{ }^{\circ} \mathrm{C}$; Detector a $300{ }^{\circ} \mathrm{C}$ - Composição da chama (30:1) (ar sintético: Hidrogênio); Gás de arraste: Nitrogênio a $1,0 \mathrm{~mL} \mathrm{~min}^{-1}$.

\section{Padrões de BTEX e curvas de referência}

Foram usados os seguintes padrões de BTEX: benzeno (SYNTY), tolueno (VETEC QF), etilbenzeno (VETEC QF) e xilenos (SYNTH). Foi preparada uma solução estoque de BTEX com a mistura dos padrões a 10,0 $\mathrm{g} \mathrm{L}^{-1}$ em metanol (grau cromatográfico, VETEC). Por diluição, a partir da solução estoque foram obtidas as seguintes soluções padrão em metanol: 1,$0 ; 0,5 ; 0,1 ; 0,01 \mathrm{e} 0,001 \mathrm{~g} \mathrm{~L}^{-1}$. Em frascos de $20 \mathrm{~mL}$ (com batoque e lacre) foram adicionados $10 \mathrm{~mL}$ de solução modificadora e 100 ì de cada padrão nas diferentes concentrações. Os frascos foram lacrados, em seguida levados ao ultrasom (THORTON, modelo T14) por 2 minutos e aquecidos em chapa de aquecimento (FANEM 315 $\mathrm{SE}$ ), por 50 minutos à temperatura de $85^{\circ} \mathrm{C}$ em banho de óleo, com agitações manuais após 10 e 30 minutos de aquecimento. Com uma seringa apropriada (gas tigth syringe - HAMILTON), devidamente aquecida, foi retirado $1 \mathrm{~mL}$ do gás proveniente do headspace e BTEX foi analisado no CG-DIC. As análises foram realizadas em triplicata.

\section{Precisão e limites de quantificação}

A precisão do método de análise cromatográfica foi determinada com injeções em triplicatas da mistura padrão de BTEX a 1 ppm, calculando os coeficientes de variação. Os limites de quantificação de cada monoaromático foram obtidos através da razão sinal: ruído de 10:1 para os componentes da mistura de BTEX. 


\section{Recuperação dos BTEX}

Para avaliar se ocorre a perda de BTEX nos frascos de headspace durante o preparo da amostra e se existe influência da textura do solo, foi determinada a recuperação (\%) de BTEX usando um solo arenoso e um argiloso. Nesse experimento foi usado em torno de 2,0000 $\mathrm{g}$ de solo (seco e triturado), transferido a um frasco de headspace de $20 \mathrm{~mL}$, em seguida adicionado $1 \mathrm{~mL}$ de solução de BTEX na concentração de $10 \mathrm{mg} \mathrm{L}^{-1}$, e imediatamente o frasco foi lacrado. As análises foram realizadas no momento e sete dias após a contaminação do solo, o experimento foi realizado em triplicata, usando-se o mesmo procedimento para o branco (solução modificadora e $1 \mathrm{~mL}$ de solução de BTEX $10 \mathrm{mg} \mathrm{L}^{-1}$ ). Até análise, os frascos foram armazenados em geladeira à temperatura de $4^{\circ} \mathrm{C} \mathrm{e}$ ao abrigo da luz. Antes da análise, foi retirado o lacre do frasco e $10 \mathrm{~mL}$ de solução modificadora foram adicionados ao frasco e este novamente lacrado. Em seguida, o frasco foi levado ao ultra-som por 2 minutos e aquecido, em chapa de aquecimento, por 50 minutos à temperatura de $85{ }^{\circ} \mathrm{C}$ em banho de óleo, com agitações manuais após 10 e 30 minutos de aquecimento. $\mathrm{O}$ banho consistiu de um refratário, com $500 \mathrm{~mL}$ de vaselina líquida e um suporte de alumínio com capacidade para 9 amostras, sobre uma chapa de aquecimento.Com uma seringa apropriada (gas tigth syringe), devidamente aquecida $\left(60^{\circ} \mathrm{C}\right)$, foram retirados $1 \mathrm{~mL}$ do gás proveniente do headspace e injetado no CG-DIC nas condições cromatográficas estabelecidas. A precisão e recuperação dos BTEX foram avaliadas determinando o coeficiente de variação e a recuperação (\%) dos BTEX através das concentrações (áreas) inicial e final.

\section{Análise de BTEX em amostras reais de solo contaminado}

Para evitar a perda dos BTEX por volatilização, a sub-amostra de solo para análise foi coletada com uma seringa (sem ponta) de 3,0 mL. Após a abertura do frasco de amostra recebido para análise de BTEX, a seringa foi rapidamente introduzida na amostra de solo para a coleta da sub-amostra a ser analisada. Comprimindo o êmbolo da seringa, cerca de 2,000 g de solo úmido foram transferidos a um frasco de penicilina (tarado) de $20 \mathrm{~mL}$, em seguida foi pesado e adicionados $10 \mathrm{~mL}$ de solução modificadora e 100 $\mu \mathrm{L}$ de metanol e o frasco imediatamente lacrado. Procedimento idêntico ao citado acima (para recuperação dos BTEX) foi realizado para a transferência dos componentes voláteis no headspace e a seguir analisado por CG-DIC. Através da área de cada componente, da equação da reta (obtida pela curva de referência em headspace) e do teor de umidade do solo, as concentrações de BTEX foram determinadas em ppm ( $\mathrm{mg} \mathrm{Kg}^{-1}$ de solo seco).

\section{Determinação da umidade do solo}

Em uma placa de Petri, devidamente tarada, foram pesados cerca de 20,00 gramas de solo. Após secagem por 24 horas em estufa a $60{ }^{\circ} \mathrm{C}$ foi transferida para um dessecador até resfriamento. Em seguida foi novamente pesada e calculada a porcentagem de umidade presente no solo.

\section{Solos usados para otimização dos métodos - características físicas e químicas}

Foram utilizados um solo argiloso (ARG), classificado como Latossolo Vermelho distroférrico coletado em uma área de cultivo de café, no IAPAR (Instituto Agronômico do Paraná) em Londrina; e outro arenoso (ARE), classificado como Latossolo Vermelho acriférrico, coletado na cidade de Jaguapitã, a $60 \mathrm{Km}$ da cidade de Londrina-PR. As amostras de solo foram coletadas a um metro da superfície do solo; as propriedades físicas e químicas são apresentadas na Tabela 1 (MOURIÑO; LOBO, 2006). 
Tabela 1. Propriedades físicas, químicas e mineralógicas dos solos arenoso (ARE) e argiloso (ARG) usados no desenvolvimento e validação do método de análise de benzeno, tolueno, etilbenzeno e xilenos.

\begin{tabular}{c|c|c}
\hline & Solo ARE & Solo ARG \\
\hline $\begin{array}{c}\text { Mineralogia \% (gibbsita, caulinita, } \\
\text { vermiculita) }\end{array}$ & $10: 50: 2$ & $6: 63: 3$ \\
\hline Granulometria (argila, silte, areia) \% & $(24 ; 1 ; 75)$ & $(72 ; 10 ; 18)$ \\
\hline Superfície específica $\left(\mathrm{m}^{2} / \mathrm{g}\right)$ & 11,7 & 85,2 \\
\hline $\mathrm{pH}$ & 3,9 & 4,4 \\
\hline Ponto de carga zero & 4,1 & 4,4 \\
\hline Valor T(CTC Total) $\left(\mathrm{cmol}_{\mathrm{d}} / \mathrm{kg}\right)$ & 7,7 & 11,9 \\
\hline Matéria Orgânica $(\% \mathrm{C})$ & 0,9 & 1,6 \\
\hline
\end{tabular}

\section{Resultados e Discussão}

Nas condições desenvolvidas por CG-DIC, foi obtida uma boa resolução para a mistura de BTEX, benzeno (tempo de retenção $=3,7 \mathrm{~min}$.), tolueno ( $\operatorname{tr}=6,4 \mathrm{~min}$.), etilbenzeno ( $\operatorname{tr}=10,4 \mathrm{~min}$.) e xilenos (o-xileno, $\operatorname{tr}=10,9$ min.; $\mathrm{me} \mathrm{p}$-xileno, $\operatorname{tr}=11,9 \mathrm{~min}$.) (Figura 3).

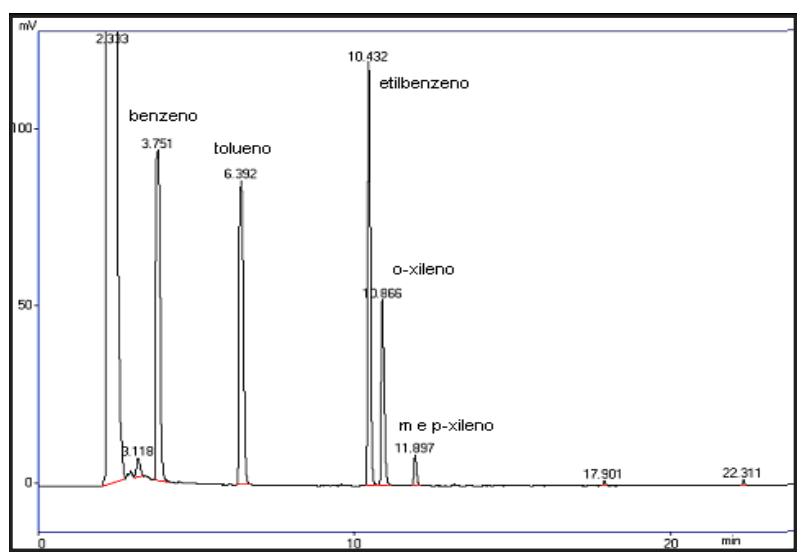

Figura 3. Cromatograma de uma mistura de BTEX a $1 \mathrm{ppm}$, por headspace e CG-DIC, coluna capilar DB1 temperatura inicial $40,0^{\circ} \mathrm{C}$ por 6 minutos e rampa de aquecimento $4,0^{\circ} \mathrm{C} / \mathrm{min}$ até 120 ${ }^{\circ} \mathrm{C} \mathrm{e} 40,0^{\circ} \mathrm{C} / \mathrm{min}$ até $200{ }^{\circ} \mathrm{C}$. Injetor a $200^{\circ} \mathrm{C}$ e detector a $300^{\circ} \mathrm{C}$.
As linearidades foram obtidas para a faixa de concentração de 0,01 a $10 \mathrm{mg} \mathrm{L}^{-1}$, apresentando coeficientes de correlação: benzeno $(\mathrm{R}=0,9892)$, tolueno $(\mathrm{R}=0,9986)$, etilbenzeno $(\mathrm{R}=0,9988)$ e xilenos $(\mathrm{R}=0,9989)$. A precisão do método de análise, avaliada através de triplicatas consecutivas da mistura padrão de BTEX a 1 ppm e obtida pelo coeficiente de variação foram: benzeno $(3,71 \%)$, tolueno $(1,47 \%)$, etilbenzeno $(2,93 \%)$ e xilenos $(1,51 \%)$. O limite de quantificação obtido, nas condições estabelecidas para o método de análise por CG - DIC e headspace, foi $0,05 \mathrm{mg} \mathrm{Kg}^{-1}$ de solo para benzeno, tolueno, etilbenzeno e xilenos. Kubinec et al. (2005) encontraram valores pouco menores para limites de quantificação de BTEX (2,0 a 3,6 $\mu \mathrm{g} \mathrm{L}$ - $\left.^{-1}\right)$ obtidos pelo método de análise por CG-DIC em amostras de água.

Com o objetivo de verificar se a textura do solo influenciava na recuperação de BTEX durante o preparo das amostras por headspace, foi determinada a recuperação desses compostos usando solos arenoso (ARE) e argiloso (ARG). Na tabela 2, são apresentados os coeficientes de variação (precisão) do método de análise CG-DIC e headspace e a recuperação (\%) de BTEX, analisadas no mesmo dia do preparo e sete dias após. Por meio da diferença entre as concentrações obtidas entre o branco e a amostra, foi calculada a recuperação (\%) dos BTEX. A concentração obtida para o branco foi considerada como a concentração inicial e a concentração obtida para a amostra, a concentração recuperada.

Tabela 2. Recuperação (\%) e coeficiente de variação (CV) do benzeno, tolueno, etilbenzeno e xilenos para os solos ARG e ARE e branco (BCO)*.

\begin{tabular}{c|c|c|c|c|c|c}
\hline & & $\begin{array}{c}\text { ARG } \\
\text { Mesmo } \\
\text { dia }\end{array}$ & $\begin{array}{c}\text { ARG } \\
7 \text { dias }\end{array}$ & $\begin{array}{c}\text { ARE } \\
\text { Mesmo } \\
\text { dia }\end{array}$ & $\begin{array}{c}\text { A RE } \\
\text { dias }\end{array}$ & $\begin{array}{c}\text { B CO } \\
\text { Mesmo } \\
\text { dia }\end{array}$ \\
\hline Benzeno & $\%$ & 100 & 76,2 & 86,9 & 77,8 & 100 \\
\hline Tolueno & C V & 10,0 & 3,0 & 4,0 & 11,9 & 7,3 \\
\hline & C V & 100 & 75,2 & 88,9 & 76,1 & 100 \\
\hline Etilbenzeno & $\%$ & 100 & 67,2 & 85,3 & 70,7 & 100 \\
\hline Xilenos & C V & 8,2 & 10,9 & 14,3 & 17,6 & 8,8 \\
\hline & C V & 100 & 65,5 & 83,7 & 73,6 & 100 \\
\hline
\end{tabular}

* ARG: argiloso, ARE: arenoso, BCO: branco 
Os coeficientes de variação obtidos para os BTEX, no mesmo dia e sete dias após a contaminação, variaram, no solo argiloso, entre a faixa de 8,1 a 10,0 e de 3,0 a 11,4, respectivamente. Os coeficientes de variação obtidos para os BTEX, no mesmo dia e sete dias após a contaminação, variaram, no solo arenoso, entre 4,0 a 18,3 e de 11,9 a 18,0 , respectivamente. Para o branco, os coeficientes de variação no mesmo dia e sete dias após a contaminação variaram na faixa de 7,3 a 10,4 e de 2,2 a 11,3 , respectivamente. Ezquerro et al. (2004) encontraram valores semelhantes para a reprodutibilidade (15\%) na metodologia de análise de BTEX em solos usando micro extração fase sólida/headspace e CG-DIC.

A recuperação dos BTEX obtida para o solo argiloso para o preparo no mesmo dia foi de $100 \%$ para todos os compostos e, após sete dias, variou de 65,5 a 76,2 \%. Para o solo arenoso, no mesmo dia, a variação foi de 83,7 a 88,9 e, após sete dias, de 70,7 a 77,8 . Por meio das \% de recuperação obtidas para o branco no mesmo dia (100\%) e após sete dias, verificou-se que houve uma diminuição na recuperação $(80,2$ a 87,7).

A recuperação dos hidrocarbonetos monoaromáticos (BTEX) nos solos arenoso e argiloso pode ser visualizada nas Figuras 4 e 5, as quais apresentam a recuperação (\%) dos BTEX para os solos argiloso e arenoso e para o branco, no mesmo dia de contaminação e sete dias após.

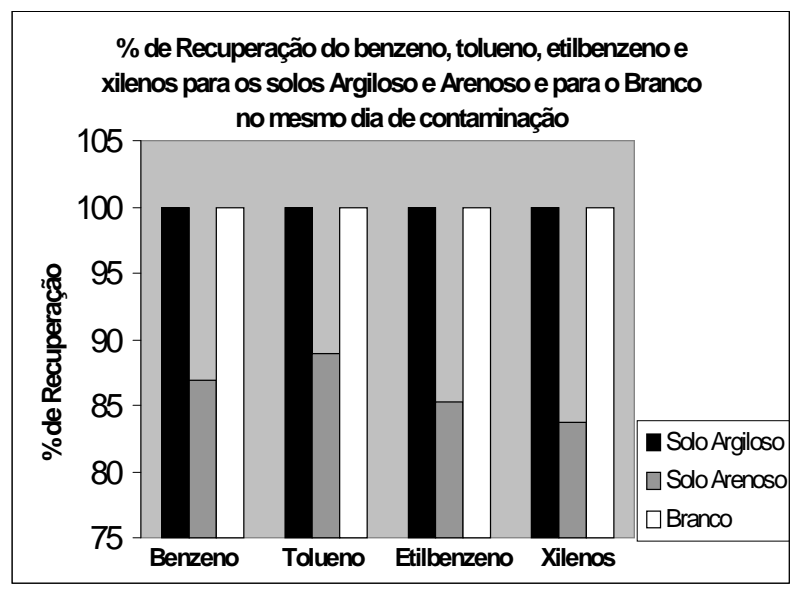

Figura 4. Recuperação (\%) de benzeno, tolueno, etilbenzeno e xilenos nos solos argiloso e arenoso e para o branco, no mesmo dia da contaminação.
Observou-se que, no mesmo dia da contaminação, a recuperação obtida para o solo arenoso foi menor, se comparada ao solo argiloso e ao branco. O solo arenoso apresenta menores teores de argila e matéria orgânica e menor CTC (capacidade de troca catiônica), quando comparado ao solo argiloso (Tabela 1). Esta menor recuperação para o solo arenoso pode ser devida à perda dos BTEX, principalmente por volatilização, já neste caso o solo apresenta maior porosidade e menor superfície quimicamente ativa, facilitando a perda dos compostos voláteis. Já o solo argiloso, apresenta maiores teores de argila e matéria orgânica, partículas que possuem capacidade de retenção dos compostos (MOURIÑO; LOBO, 2006), não apresentou perda dos BTEX na análise realizada no mesmo dia da contaminação.

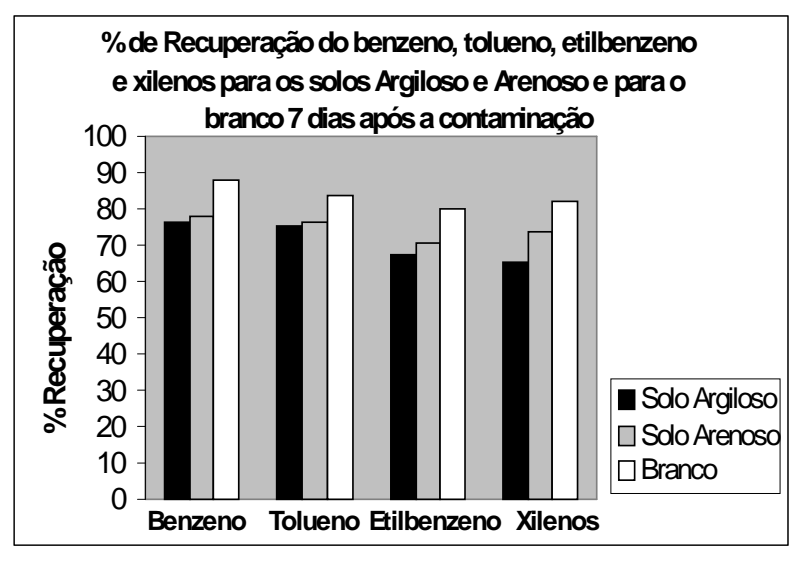

Figura 5. Recuperação (\%)de benzeno, tolueno, etilbenzeno e xilenos nos solos argiloso e arenoso e para o branco, após sete dias de contaminação.

A análise realizada sete dias após a contaminação apresentou menor recuperação tanto para o solo arenoso como para o argiloso, quando comparada ao branco e também àquela realizada no mesmo dia da contaminação. A recuperação menor que $100 \%$ apresentada pelo branco após sete dias também indica que ocorre a perda dos compostos voláteis (BTEX) por meio do lacre, mesmo na ausência do solo, demonstrando a ocorrência da volatilização durante a estocagem. 


\section{Conclusão}

A análise usando headspace estático é rápida (50 minutos), adaptável para múltiplas amostras, de baixo custo e elimina o uso de solventes orgânicos. O método de análise cromatográfica mostrou boa resolução, em um baixo tempo de análise (menos de 30 minutos). Os limite de quantificação $\left(0,05 \mathrm{mg} \mathrm{Kg}^{-1}\right.$ solo) para benzeno, tolueno, etilbenzeno e xilenos encontram-se abaixo dos valores orientadores, que variam de 0,15 a $95 \mathrm{mg} \mathrm{Kg}^{-1}$ solo (Valores orientadores, 2005) estabelecidos para determinar a qualidade do solo (uso industrial). Isso permite a aplicação deste método na análise de BTEX em amostras reais de solos para a identificação de passivo ambiental de postos de combustíveis. O preparo da amostra no frasco de headspace e a posterior análise cromatográfica devem ser realizados no mesmo dia, para evitar a perda dos compostos e consequentemente para que os resultados sejam confiáveis para avaliação segura de passivos ambientais. A recuperação de BTEX depende da textura do solo, é menor em solos arenosos, mas ainda com boa recuperação $(83,7$ a 88,9\%), e tem aceitável precisão para análise desses compostos voláteis em solos contaminados com derivados de petróleo.

\section{Referências}

ARAMBARRI, I.; LASA, M.; GARCIA, R.; MILLAN, E. Determination of fuel dialkyl ethers and BTEX in water using headspace solid-phase microextraction and gas chromatography-flame ionization detection. Journal of Chromatography A, Amsterdam, v.1033, n.2, p.193-203, apr. 2004.

COMPANHIA DE TECNOLOGIA DE SANEAMENTO AMBIENTAL-CETESB. Decisão de Diretoria n. ${ }^{\circ}$ 195-2005- E, de 23 de novembro de 2005. Disponível em: http:// www.cetesb.sp.gov.br/solos/relatorios/ tabela_valores_2005.pdf. Acesso em: 17 Ago. 2006
EZQUERRO, O.; TENA, M.; ORTIZ, G.; PONS, B. Determination of BTEX in soils by multiple headspace solid-phase microextraction. Journal of Chromatography A, Amsterdam, v.1035, n.1, p.1722, apr. 2004.

FINE, P., GRABER, E. R.; YARON, B. Soil interactions with petroleum hydrocarbons: Abiotic processes. Soil Technology, Amsterdam, v.10, n.2, p.133-153, feb. 1997.

KUBINEC, R.; ADAMUSCIN, J.; JURDÁKOVÁ, H.; FOLTIN, M.; OSTROVSKÝ, I.; KRAUS, A.; SOJÁK, L. Gas chromatographic determination of benzene, toluene, ethylbenzene, xylenes using flame ionization detector in water samples with direct aqueous injection up to $250 \mu \mathrm{l}$. Journal of Chromatography A, Amsterdam, v.1084, n.1/2, p.9094, aug. 2005.

MARTINEZ, E.; LACORTE, S.; LLOBET, I.; VIANA, P.; BARCELÓ, D. Multicomponent analysis of volatile organic compounds in water by automated purge and trap coupled to gás chromatography-mass spectrometry. Journal of Chromatography A, Amsterdam, v.959, n.1/2, p.181190, jun. 2002.

MOHAMMADI, A.; ALIZADEH, N. Automated dynamic headspace organic solvent film microextraction for benzene, toluene, ethylbenzene and xylene Renewable liquid film as a sampler by a programmable motor. Journal of Chromatography A, Amsterdam, v.1107, n.1/2, p.19-28, feb. 2006.

MOURIÑO, R. O.; LOBO, I. Adsorção de herbicidas iônicos (2,4-D e atrazina) em Latossolos Brasileiros. CONGRESO IBEROAMERICANO DE FÍSICA Y QUÍMICA AMBIENTAL, 4., 2006, Badajoz (Espanha). Anais... Badajoz: SiFyQA, 2006. p.325332. 
RESTEK CORPORATION. A Technical Guide for Static Headspace Analysis Using GC. Disponível em: <http://www.restekcorp.com/restek/images/ external/59895A.pdf $>$. Acesso em: 9 out. 2004.

SEMPLE, K. T.; REID, B. J.; FERMOR, T. R. Impact of composting strategies on the treatment of soils contaminated with organic pollutants. Environmental Pollution, v.112, n.2, p.269-283, 2001. 